El-Hage W, Gaillard P. Incidence clinique des psychotraumatismes dans la consultation externe d'un service de psychiatrie. Annales Médico-Psychologiques 2003;161(10):743-748. doi:10.1016/S0003-4487(03)00161-6

\title{
Incidence clinique des psychotraumatismes dans la consultation externe d'un service de psychiatrie
}

\section{Clinical impact of psychotraumas in outpatients' department of psychiatry}

\author{
W. El-Hage ${ }^{1}$, P. Gaillard ${ }^{2}$ \\ ${ }^{1}$ EA3248 Psychobiologie des Emotions et IFR 120, Clinique Psychiatrique Universitaire, CHU \\ de Tours, 37044 Tours, France
}

${ }^{2}$ Clinique Psychiatrique Universitaire, CHU de Tours, 37044 Tours, France

Titre abrégé: Aspects cliniques psychotraumatiques

Correspondance: Wissam El-Hage, EA3248 Psychobiologie des Emotions et IFR 120, Clinique Psychiatrique Universitaire, CHU de Tours, 37044 Tours, France

\begin{abstract}
Résumé - Les auteurs ont cherché à évaluer l'impact des psychotraumatismes dans une population de consultants en psychiatrie. Cent quarante patients psychiatriques ambulatoires ont été inclus successivement sans distinction de leur pathologie psychiatrique. Ils ont rempli un inventaire d'évènements vécus potentiellement traumatiques, une échelle des expériences dissociatives psychiques, un questionnaire des expériences dissociatives somatoformes, et ont eu un entretien structuré pour le diagnostic du «trouble de stress post-traumatique» (selon le DSM-IV). Cent sujets ont vécu des traumatismes psychiques, et 76 patients ont éprouvé un trouble de stress post-traumatique. Les troubles dissociatifs retrouvés sont significativement corrélés au vécu traumatique et au trouble de stress post-traumatique. L'impact traumatique est trouvé d'autant plus sévère que le nombre d'évènements vécus est élevé. Cette étude confirme la prévalence élevée des traumatismes psychiques et des séquelles psychotraumatiques retrouvées dans la consultation externe d'un service de psychiatrie, indépendamment du trouble mental pris en charge.
\end{abstract}

Mots clés: Trauma / trouble de stress post-traumatique / troubles dissociatifs

\begin{abstract}
Summary - Clinical impact of psychotraumas in outpatients' department of psychiatry. The authors aimed at evaluating the impact of psychotraumas in psychiatric outpatients. One hundred forty psychiatric outpatients were consecutively included, without distinction of their current pathology. They filled in an inventory of potentially traumatic experiences, a psychological dissociative experiences scale (DES), a somatoform dissociative experiences questionnaire (SDQ-20), and underwent a structured interview for diagnosis of posttraumatic stress disorder (CAPS, according to the DSM-IV). One hundred subjects related psychological trauma, and 76 patients suffered from posttraumatic stress disorder. Dissociative disorders are found significantly correlated to traumatic experience and to posttraumatic stress disorder. The traumatic impact is found more substantial as the number of events lived was elevated. This study confirms the elevated prevalence of the psychological traumas and psychotraumatic aftermaths in outpatients of a department of psychiatry, independently from the mental disorder. It confirms the strong association between trauma and dissociative symptoms as a whole, including somatoform dissociation.
\end{abstract}

Key words: Trauma / posttraumatic stress disorder / dissociative disorders 
Notre travail a pour objectif l'étude de la relation entre des traumatismes psychiques et leur incidence clinique dans une population ambulatoire en psychiatrie. La population des patients psychiatriques ambulatoires comporte un nombre élevé (18\%) de patients souffrant d'une symptomatologie psychotraumatique [5]. Certains auteurs [12] trouvent chez 70\% des patientes suivies en consultation externe de psychiatrie des antécédents d'abus sexuels. Dans une enquête auprès de 90 femmes atteintes de schizophrénie ou de trouble bipolaire, les agressions sexuelles sont trouvées plus fréquentes qu'en population générale [7]. De plus, chez les femmes atteintes de schizophrénie, le viol est associé avec une plus grande sévérité de leur trouble. Dans une étude portant sur 181 patients psychiatriques ambulatoires, Switzer et ses collègues [19] découvrent que $94 \%$ de ces patients ont vécu une expérience traumatique dans leur vie, parmi lesquels $42 \%$ souffrent d'un trouble de stress post-traumatique (PTSD) chronique. Les antécédents psychiatriques, personnels ou familiaux, semblent augmenter le risque d'exposition au trauma, et le développement d'un PTSD en cas de vécu traumatique [10]. De plus vivre une expérience traumatique et éprouver une symptomatologie psychotraumatique complique les processus de récupération de tout autre trouble mental [13]. Les antécédents d'abus sexuels dans l'enfance accroissent le risque de psychopathologie, sans être impliqués dans l'émergence de troubles psychiatriques spécifiques [6]. De la même façon les abus physiques de l'adolescent semblent représenter un facteur de risque significatif pour le développement de nombreux troubles psychiatriques qu'ils soient spécifiquement post-traumatiques (PTSD) ou moins spécifiques de l'étiologie traumatique (dépression majeure, dysthymie, troubles des conduites, abus de drogues et tabagisme) [11]. Breslau et ses collaborateurs [4] ont identifié chez les sujets souffrant de PTSD chroniques un nombre plus élevé de symptômes de PTSD et une comorbidité plus élevée par rapport aux PTSD non chroniques (des troubles anxieux, des troubles de l'humeur, ou l'abus en substances psychoactives).

La symptomatologie post-traumatique est variable, et ne se limite pas au seul PTSD. Les symptômes dissociatifs (12\% en population psychiatrique ambulatoire [18], mais cette symptomatologie reste souvent méconnue des cliniciens) sont trouvés souvent associés aux antécédents de traumatisme psychique (chez l'enfant ou l'adulte), ainsi qu'au trouble somatisation [15] et au trouble de conversion. Le PTSD, la dissociation, la somatisation et la perturbation des affects représentent une forme d'adaptation au traumatisme [20].

De cette courte revue de travaux antérieurs nous retenons, du fait de l'association nette entre exposition à des traumatismes psychiques et demande de soins médicaux, l'importance de repérer les patients avec antécédents traumatiques et PTSD afin de proposer une prise en charge adaptée.

\section{MATERIEL ET METHODE}

\section{Sujets}

Les sujets de l'étude étaient suivis en consultation externe à la Clinique Psychiatrique du Centre Hospitalo-Universitaire de Tours. Le motif de consultation relevait de pathologies psychiatriques diverses. Le diagnostic psychiatrique principal n'a pas été recherché. L'étude a donc été proposée à tous les patients ambulatoires. Après explication des objectifs, des modalités de l'entretien et de remplissage des différentes échelles, de façon claire et simple, nous avons recueilli le consentement du sujet. Cent soixante-deux sujets ont été sollicités, 9 sujets ont refusé de participer, 140 sujets ont participé à notre étude, et 13 sujets n'ont pas pu être inclus, pour des raisons diverses: dysfonctionnement cognitif (3), étrangers maîtrisant peu le français (3), analphabétisme (3), patients gravement malades, par exemple troubles dépressifs sévères (2), états d'agitation aiguë (2). Le comité d'éthique de Tours a émis un avis favorable au déroulement de cette étude. 


\section{Instruments d'étude}

La CAPS (Clinician-Administered Post-traumatic stress disorder Scale for DSM-IV) est un instrument diagnostique catégoriel du PTSD (actuel, et par le passé), suivant un entretien clinique structuré, utilisant des questions standardisées brèves et explicites, et une échelle quantitative de sévérité du trouble, développée par Blake et ses collègues [4]. Cet outil de référence largement utilisé [22] possède d'excellentes qualités psychométriques. La CAPS contient une liste exhaustive des évènements potentiellement traumatiques selon la définition du critère A du PTSD du DSM-IV. Elle permet une évaluation détaillée et complète de chacun des symptômes du PTSD du DSM-IV pour leur fréquence (de 0 à 4 ) et leur intensité (de 0 à 4 ), actuelles et passées. Elle permet de faire le diagnostic si les clusters A (événement traumatique), $\mathrm{E}$ (durée supérieure à un mois) et $\mathrm{F}$ (souffrance clinique) sont remplis, avec au moins 1 item du cluster B (symptômes de reviviscence), 3 items du cluster C (symptômes d'évitement et d'émoussement), et 2 items du cluster D (symptômes d'hypervigilance). Un score total est obtenu par addition des sous-scores des clusters B, C et D. La CAPS peut être utilisée par des cliniciens expérimentés, ou bien des non professionnels formés et entraînés. La version française de la CAPS a été publiée par un laboratoire pharmaceutique dans le cadre d'une étude multicentrique.

La DES (Dissociative Experiences Scale) est un auto-questionnaire de 28 items, développé par Bernstein et Putnam [1] à partir de l'expérience clinique, afin de permettre une mesure valide de la dissociation dans des populations symptomatiques ou non. C'est une échelle analogique visuelle. Le sujet signale si l'expérience mentionnée par la question lui arrive et quantifie en marquant avec un trait vertical une ligne allant de $0 \%$ (jamais) à 100\% (tout le temps). On obtient un score pouvant aller de 0 à 100 . Son usage est simple et rapide. De nombreuses études de validation et d'analyse de la structure de l'échelle ont été réalisées en langue anglaise $[1,2]$ et en langue française [8].

Le SDQ (Somatoform Dissociation Questionnaire) est un auto-questionnaire de 20 items développé par Nijenhuis et ses collègues [14] à partir d'un groupe de 77 items fondés sur des symptômes cliniques somatoformes, non expliqués médicalement, présents lors de troubles dissociatifs du DSM-IV. Vingt items ont été retenus à l'aide d'analyses de régression logistique, relevant l'aptitude de l'échelle à discriminer les sujets souffrant de troubles dissociatifs d'un groupe contrôle sans trouble dissociatif. Les items sont cotés de 1 à 5 . Le sujet doit choisir, pour chacun des items, une réponse: cela ne me concerne pas du tout (1), cela s'applique à moi un peu (2), modérément (3), nettement (4), extrêmement (5). Le score peut varier entre 20 et 100. Le SDQ a été validé aux Pays-Bas [16], en Turquie [17], partiellement au Royaume-Uni [21], ainsi qu'en France [9]. Le SDQ est une échelle de bonnes qualités psychométriques qui a été développée pour quantifier les phénomènes de dissociation somatoforme, c'est-à-dire de conversion.

\section{Procédure}

L'étude a été réalisée par un seul cotateur clinicien entraîné à l'utilisation des échelles. Les 140 sujets retenus ont remplis les questionnaires au cours d'un seul et même entretien, après la consultation habituelle. L'enquête garantit l'anonymat et comporte des éléments socio-démographiques. Dans un premier temps le sujet a été interrogé par l'expérimentateur afin de répondre à la CAPS. Immédiatement après, le sujet remplissait le SDQ et la DES. L'expérimentateur vérifiait ensuite le remplissage entier des questionnaires.

\section{Analyses statistiques}

Les analyses des données de cette étude ont porté sur les 140 patients inclus. Les variables quantitatives sont décrites à l'aide de moyennes, écart-types et étendues. Les comparaisons de variables continues entre deux groupes ont été réalisées à l'aide de comparaisons de moyennes 
utilisant le test de Student, ou bien une analyse de variance lors d'ajustement de ces variables à des covariantes (âge, sexe). Les tests $t$ ont été achevés pour examiner les scores de la DES et du SDQ parmi les patients. L'association entre plusieurs variables continues a été étudiée en calculant des coefficients de corrélation. Les associations entre les scores de la CAPS, de la DES, et du SDQ ont été calculées à l'aide du coefficient de corrélation de Pearson. L'ajustement de ces résultats sur des covariantes (âge, sexe) a été réalisé à l'aide d'un type d'analyse de variance utilisant le test $F$. Les données ont été exploitées en utilisant le logiciel d'analyses statistiques SYSTAT SPSS.

\section{RESULTATS \\ Description de l'échantillon}

La moyenne d'âge des 140 sujets de l'étude est de 40.4 ans $( \pm 14.1,17-76)$. La moyenne d'âge des sujets féminins est de 39.7 ans $( \pm 13.1)$, celle des sujets masculins de $41.4( \pm 15.4)$. Soixante et un sujets (44\%) sont de sexe masculin. Il n'y a pas de différence significative entre les âges moyens des hommes et des femmes de cet échantillon $(\mathrm{t}=-0.727, \mathrm{p}=0.469)$.

\section{Les expériences traumatiques}

Dans notre échantillon, nous dénombrons 116 sujets ayant vécu 491 expériences potentiellement traumatiques, 100 sujets ayant vécu des expériences traumatiques au sens du critère A du DSM-IV (tableau I). L'impact traumatique de ces expériences est variable selon la catégorie d'expérience. Ainsi le critère A2 (réaction de peur intense, d'impuissance ou d'horreur) est rempli dans: $52 \%$ des catastrophes naturelles, $76 \%$ des accidents de travail ou domestiques graves, $79 \%$ des agressions physiques, $90 \%$ des agressions physiques avec une arme, $100 \%$ des agressions sexuelles, $80 \%$ des cas de captivité, et $60 \%$ des cas de souffrance humaine grave.

De point de vue épidémiologique il est intéressant de savoir si le développement de troubles post-traumatiques peut être lié au nombre d'évènements vécus ou bien s'agit-il d'une spécificité évènementielle? Dans notre échantillon, indépendamment du type d'événement vécu, plus le nombre d'évènements vécus est élevé plus le risque de développer un PTSD est élevé. Ainsi ce risque est de $8.3 \%$ en cas d'un seul événement vécu, et devient supérieur à $60 \%$ à partir de 4 évènements. Parallèlement les scores aux différentes échelles augmentent de façon quasi-linéaire avec le nombre d'évènements vécus (tableau II). Nous trouvons des corrélations significatives $(\mathrm{ddl}=138, \mathrm{p}<0.0001)$ entre le nombre d'évènements vécus et les scores moyens à la CAPS ( $\mathrm{r}=0.512)$, à la DES ( $\mathrm{r}=0.577)$, et au SDQ ( $\mathrm{r}=0.43)$.

Nous avons voulu ensuite étudié la liaison entre les scores moyens des différents instruments de l'étude et le type d'événements vécus. Les scores moyens les plus élevés à la CAPS (>45) sont trouvés chez les patients ayant vécu: agression physique, agression avec une arme, agression sexuelle, autre expérience sexuelle pénible, ou mort violente de quelqu'un de l'entourage (homicide, suicide). Les scores moyens les plus élevés à la DES (>22) sont trouvés chez les patients ayant vécu: agression avec une arme, agression sexuelle, autre expérience sexuelle pénible, captivité, ou mort violente (homicide, suicide) de quelqu'un de l'entourage. Les scores moyens les plus élevés au SDQ (>31) sont trouvés chez les patients ayant vécu: catastrophe naturelle, exposition à une substance toxique dangereuse, agression sexuelle, autre expérience sexuelle pénible, ou captivité. Ainsi les scores moyens les plus élevés aux différents instruments de l'étude (CAPS, DES, SDQ) sont trouvés chez les patients ayant vécu soit une agression sexuelle soit une autre expérience sexuelle pénible. En effet, parmi les 44 patients victimes d'agressions sexuelles ( $\geq 1$ événement par sujet) nous identifions 30 sujets avec un diagnostic de PTSD actuel (score moyen 51.34), et 31 sujets avec un diagnostic de PTSD par le passé (score moyen 49.82) à la CAPS. Ces 44 sujets ont des scores moyens de 23.1 à la DES, et de 32.3 au SDQ. 
Pour faire le lien entre la clinique post-traumatique et le type d'événement vécu, nous avons réalisé une analyse de variance (test $F$ ) des scores moyens de la CAPS, de la DES, et du SDQ en liaison avec le type d'événements vécus (tableau III). Ainsi certains évènements sont en liaison avec des scores moyens significativement plus élevés aux trois instruments de l'étude: exposition à une substance toxique dangereuse, agression physique, agression sexuelle, autre expérience sexuelle pénible, captivité, souffrance humaine grave.

\section{Analyse des symptômes du PTSD selon le DSM-IV}

Nous avons évalué la fréquence de survenue des symptômes du PTSD (actuel ou par le passé) chez les sujets ayant vécu des expériences traumatiques, à partir des items de la CAPS. Un item est considéré positif si le symptôme est survenu au moins une fois (fréquence=1) et avec une intensité au moins minimale (intensité=2). Ainsi, les items les plus fréquemment retrouvés sont les souvenirs répétitifs et envahissants et les symptômes d'angoisse psychologique et physiologique lors de rappels traumatiques (items du cluster «Reviviscence»); l'évitement des pensées, des sensations ou des conversations et le sentiment de détachement ou d'étrangeté (items du cluster «Evitement et d'émoussement»); la difficulté à s'endormir ou à rester endormi(e) (item du cluster «Hypervigilance»). Si nous calculons les différences entre la proportion de patients présentant un symptôme particulier parmi les critères diagnostiques du PTSD (actuel) du DSM-IV par catégorie d'évènements potentiellement traumatiques vécus (à l'aide du test de Kruskal-Wallis), nous trouvons que ces différences ne sont pas statistiquement significatives pour le cluster $B(H=6.074, p=0.19)$ et le cluster $E(H=6.982, p=0.14)$, et sont statistiquement significatives pour le cluster $\mathrm{C}$ «Evitement et émoussement» $(\mathrm{H}=13.745$, $\mathrm{p}<0.01)$ le cluster $\mathrm{D}$ «Hypervigilance» $(\mathrm{H}=491, \mathrm{p}<0.05)$, et le cluster $\mathrm{F}$ «Souffrance clinique» $(\mathrm{H}=11.5, \mathrm{p}<0.05)$. Nous pouvons conclure que les symptômes les plus fréquents, et peut-être les plus spécifiques du PTSD, sont ceux de la répétition traumatique cognitive (cluster B).

\section{Pathologies post-traumatiques}

Parmi le groupe de sujets aux antécédents d'expériences traumatiques, nous dénombrons 62 sujets avec un PTSD actuel, et 57 sujets avec un PTSD par le passé. Dans leur vie $54.3 \%$ des sujets de cet échantillon ont éprouvé un PTSD $(n=76)$.

Les scores à la DES s'étendent de 0 à $65.9(\mathrm{M}=14.6, \mathrm{ET}=12.9)$. Le score moyen à la DES est de 18.6 chez les sujets aux antécédents d'expériences potentiellement traumatiques, significativement différent de 4.8 chez les sujets sans antécédent similaire $(\mathrm{t}=5.981, \mathrm{ddl}=138$, $\mathrm{p}<0.001)$. Les scores au SDQ s'étendent de 20 à $76(\mathrm{M}=27.1, \mathrm{ET}=8.76)$. Le score moyen au SDQ est de 29.5 chez les sujets aux antécédents d'expériences potentiellement traumatiques, significativement différent de 21.0 chez les sujets sans antécédent similaire $(\mathrm{t}=5.725, \mathrm{ddl}=138$, $\mathrm{p}<0.001$ ). Les scores moyens à la DES et au SDQ en fonction du diagnostic de PTSD (actuel et par le passé) sont présentés dans le tableau IV.

Les études de corrélation réalisées sur notre échantillon mettent en évidence certaines associations entre trauma et dissociation. La dissociation à expression somatique est significativement $(\mathrm{p}<0.01)$ corrélée au nombre d'évènements vécus $(\mathrm{r}=0.413)$, aux «accidents» $(\mathrm{r}=0.202)$, aux «agressions physiques ou sexuelles» $(\mathrm{r}=0.487)$, et aux «traumatismes liés à la maladie» $(\mathrm{r}=0.225)$. Les individus avec un PTSD actuel $(\mathrm{t}=6.990)$ ou par le passé $(\mathrm{t}=5.774)$ ont des scores significativement $(\mathrm{ddl}=138, \mathrm{p}<0.001)$ plus élevés au SDQ que les individus sans PTSD. Après ajustement de ces résultats sur le sexe, nous trouvons toujours une significativité statistique $(\mathrm{ddl}=136, \mathrm{p}<0.001)$ entre les scores au SDQ et l'existence de PTSD actuel $(\mathrm{F}=21.366)$ ou par le passé $(\mathrm{F}=13.014)$. Les individus avec un PTSD actuel $(\mathrm{t}=7.682)$ ou par le passé $(\mathrm{t}=5.410)$ ont des scores significativement $(\mathrm{ddl}=138, \mathrm{p}<0.001)$ plus élevés à la DES que les individus sans PTSD. 


\section{DISCUSSION}

Cette étude confirme la prévalence élevée des traumatismes psychologiques et des séquelles psychotraumatiques dans la consultation externe d'un service psychiatrique universitaire, indépendamment du motif de la consultation ou du trouble mental pris en charge. Les événements les plus fréquents sont ceux en rapport direct avec l'altération de la santé suivis des agressions physiques ou sexuelles. Toutefois les évènements avec le plus grand impact traumatique sont les agressions sexuelles, les agressions physiques avec une arme, les autres agressions physiques. Ces évènements sont ceux où le sujet est directement confronté à une effraction de l'enveloppe corporelle et à une réelle confrontation à la mort. L'impact traumatique est trouvé d'autant plus sévère que le nombre d'évènements vécus est élevé. Cette constatation pose la question d'un modèle de vulnérabilité acquise, et dessine les limites des résiliences individuelles face à des traumatismes répétés. Ainsi, tout sujet victime de traumatismes répétés risque de développer une pathologie post-traumatique indépendamment de sa personnalité antérieure. Par ailleurs les scores moyens les plus élevés aux différents instruments de l'étude sont trouvés chez les patients ayant vécu une agression sexuelle. Autrement dit vivre une agression sexuelle est trouvé significativement lié au développement d'un PTSD et de troubles dissociatifs. De plus, les victimes (non étayées par le groupe d'appartenance familial ou social) d'agressions sexuelles restent le plus souvent dans le secret et dans la souffrance contrairement aux autres évènements où le traumatisme est habituellement porté à la connaissance collective et donc suscite des réactions de soutien ou de sympathie.

Les symptômes du PTSD du DSM-IV retrouvés de façon constante, et peut-être les plus spécifiques du PTSD, sont ceux de la répétition traumatique cognitive. Cette hypermnésie de souvenirs traumatiques est parfois associée à une amnésie psychogène (dans plus d'un tiers des cas de PTSD). Des études sont nécessaires afin de déterminer dès la phase péritraumatique le type du trouble, et évaluer les capacités d'encodage, de stockage et de rappel au long terme.

Cet échantillon comporte un nombre élevé de syndromes post-traumatiques partiellement dus à l'existence d'une consultation spécialisée dans le traitement des syndromes psychotraumatiques. Treize sujets n'ont pas été inclus dans notre étude pour des raisons diverses signalées dans les résultats. Ce nombre s'explique par la durée de l'entretien, la passation d'une batterie importante d'instruments, et la durée nécessaire pour obtenir des informations précises, ce qui peut rebuter certaines personnes. Toutefois, un vif intérêt pour ces instruments cliniques a été manifesté par les sujets inclus qui y trouvaient un reflet objectif de leur vécu subjectif. Le maniement de la CAPS nécessite une certaine vigilance clinique: un temps d'arrêt est nécessaire du fait de l'émergence de manifestations d'angoisse liées à des rappels traumatiques. Les auto-questionnaires (DES, SDQ) sont relativement faciles à appliquer et à exploiter.

\section{CONCLUSION}

Le traumatisme psychologique provoque une symptomatologie clinique spécifique, allant des états de stress aigu aux troubles de la personnalité, et complique la prise en charge des patients vulnérables. Un acte simple de dépistage lors de toute consultation en psychiatrie consisterait à répertorier le nombre et le type d'évènements potentiellement traumatiques vécus au cours de la vie. Cet acte peut orienter la consultation vers le diagnostic aisé d'états de stress post-traumatiques, de troubles dissociatifs, psychiques et somatoformes, trop souvent ignorés. L'identification par le thérapeute de cette problématique est déjà un début de thérapeutique.

\section{REMERCIEMENTS}

Remerciements à Monsieur le Professeur Pierre Sizaret pour sa relecture du manuscrit. 


\section{REFERENCES}

1 Bernstein E, Putnam FW. Development, reliability, and validity of a dissociation scale. J Nerv Ment Dis 1986;174(12):727-735.

2 Bernstein E, Putnam FW, Ross CA, et al. Validity of the dissociative experiences scale in screening for multiple personality disorder: a multicenter study. Am J Psychiatry 1993;150:1030-1036.

3 Blake DD, Weathers FW, Nagy LM, et al. A clinician rating scale for assessing current and lifetime PTSD: The CAPS-1. Behav Ther 1990;13:187-188.

4 Breslau N, Davis GC. Posttraumatic stress disorder in an urban population of young adults: risk factors for chronicity. Am J Psychiatry 1992;149:671-675.

5 Brom D, Witztum E. Recent trauma in psychiatric outpatients. Am J Orthopsychiatry 1992;62(4):545-551.

6 Bulik CM, Prescott CA, Kendler KS. Features of childhood sexual abuse and the development of psychiatric and substance use disorders. Br J Psychiatry 2001;179:444-449.

7 Darves-Bornoz JM. Syndromes traumatiques du viol et de l'inceste. Rapport de la LXXXXIV ${ }^{\circ}$ session du congrès de psychiatrie et de neurologie de langue française. Paris: Masson, 1996.

8 Darves-Bornoz JM, Degiovanni A, Gaillard P. Validation of a French version of the Dissociative Experiences Scale in a rape victim population. Can J Psychiatry 1999;44:271-275.

9 El-Hage W, Darves-Bornoz JM, Allilaire JF, et al. Posttraumatic somatoform dissociation in French psychiatric outpatients. Journal of Trauma \& Dissociation 2002;3(3):59-74.

10 Hidalgo RB, Davidson JRT. Posttraumatic stress disorder: epidemiology and health-related considerations. J Clin Psychiatry 2000;61(S7):5-13.

11 Kaplan SJ, Pelcovitz D, Salzinger S, et al. Adolescent physical abuse: risk for adolescent psychiatric disorders. Am J Psychiatry 1998;155:954-959.

12 Lombardo S, Pohl R. Sexual abuse history of women in a psychiatric outpatient clinic. Psychiatric Services (Washington, D.C.) 1997;48(4):534-536.

13 McFarlane AC, Bookless C, Air T. Posttraumatic stress disorder in a general psychiatric inpatient population. J Trauma Stress 2001;14(4):633-645.

14 Nijenhuis ERS, Spinhoven P, Van Dyck R, et al. The development and psychometric characteristics of the Somatoform Dissociation Questionnaire (SDQ-20). J Nerv Ment Dis 1996;184(11):688-94.

15 Pribor EF, Yutzy SH, Dean JT, et al. Briquet's syndrome, dissociation and abuse. Am J Psychiatry 1993;150:1507-1511.

16 Ross CA, Joshi S, Currie R. Dissociative experiences in the general population. Am J Psychiatry 1990;147:1547-1552.

17 Sar V, Kundakci T, Kiziltan E, et al. Differentiating dissociative disorders from other diagnostic groups through somatoform dissociation in Turkey. Journal of Trauma \& Dissociation 2000;1(4):67-80.

18 Sar V, Tutkun H, Alyanak B, et al. Frequency of dissociative disorders among psychiatric outpatients in Turkey. Compr Psychiatry 2000;41(3):216-222.

19 Switzer GE, Dew MA, Thompson K, et al. Posttraumatic stress disorder and service utilization among urban mental health center clients. J Trauma Stress 1999;12(1):25-39.

20 Van der Kolk BA, Pelcovitz D, Roth S, et al. Dissociation, somatization, and affect dysregulation: the complexity of adaptation of trauma. Am J Psychiatry 1996;153:83-93.

21 Waller G, Hamilton K, Elliott P, et al. Somatoform dissociation, psychological dissociation and specific forms of trauma. Journal of Trauma \& Dissociation 2000;1(4):81-98.

22 Weathers FW, Keane TM, Davidson JR. Clinician-administered PTSD scale: a review of the first ten years of research. Depress Anxiety 2001;13(3):132-156. 
Tableau I. Prévalence des expériences potentiellement traumatiques rapportées chez les patients psychiatriques ambulatoires $(\mathrm{n}=116)$ ayant vécu au moins une expérience potentiellement traumatique

\begin{tabular}{|c|c|c|c|}
\hline & Evènements traumatiques & (491) & $\%$ \\
\hline & Catastrophes & 53 & 10.8 \\
\hline 1. & Catastrophe naturelle (inondation, tremblement de terre, tornade, ouragan) & 23 & 4.7 \\
\hline 2. & Incendie ou explosion & 30 & 6.1 \\
\hline & Accidents & 79 & 16.1 \\
\hline 3. & Accident de transport & 50 & 10.2 \\
\hline 4. & $\begin{array}{l}\text { Accident de travail ou domestique graves (brûlure, chute avce fracture, } \\
\text { explosion, coupure, écrasement) }\end{array}$ & 25 & 5.1 \\
\hline 5. & Exposition à une substance toxique dangereuse & 4 & 0.8 \\
\hline & Agressions physiques ou sexuelles & 157 & 32.0 \\
\hline 6. & $\begin{array}{l}\text { Agression physique (maltraitance physique dans l'enfance, violences de } \\
\text { couple, dans lieux publics avec ou sans vol) }\end{array}$ & 54 & 11.0 \\
\hline 7. & Agression avec une arme (arme blanche, arme à feu) & 41 & 8.4 \\
\hline 8. & $\begin{array}{l}\text { Agression sexuelle (viols uniques ou répétés, dans l'enfance ou à l'âge adulte, } \\
\text { tentative de viol) }\end{array}$ & 36 & 7.3 \\
\hline 9. & $\begin{array}{l}\text { Autre 'expérience' sexuelle pénible, commise contre son gré (viol par le } \\
\text { conjoint, attouchements, tentative de viol) }\end{array}$ & 26 & 5.3 \\
\hline & Faits de guerre et faits assimilés & 21 & 4.3 \\
\hline 10. & Combat ou exposition à une zone en guerre & 16 & 3.3 \\
\hline 11. & Captivité (séquestration à domicile, séquestration à l'étranger) & 5 & 1.0 \\
\hline & Traumatismes en rapport direct avec l'altération de la santé & 181 & 36.8 \\
\hline 12. & Maladie ou blessure menaçant la survie & 30 & 6.1 \\
\hline 13. & Souffrance humaine grave (maltraitance psychique, due à une maladie) & 48 & 9.9 \\
\hline 14. & Mort soudaine, violente & 28 & 5.7 \\
\hline 15. & Mort soudaine, inattendue d'un proche & 68 & 13.7 \\
\hline 16. & Blessure grave, dommage important ou mort causés à quelqu'un d'autre & 7 & 14 \\
\hline
\end{tabular}


Tableau II. Description quantitative de la clinique post-traumatique en relation avec le nombre d'événements vécus potentiellement traumatiques.

\begin{tabular}{ccccccc}
\hline \multicolumn{2}{c}{ Nombre } & Critère A & \multicolumn{2}{c}{ PTSD actuel } & \multicolumn{2}{c}{ Troubles dissociatifs } \\
\hline Evènements & Sujets & rempli & Nombre & $\begin{array}{c}\text { Scores moyens } \\
\text { CAPS }\end{array}$ & $\begin{array}{c}\text { Scores moyens } \\
\text { DES }\end{array}$ & $\begin{array}{c}\text { Scores moyens } \\
\text { SDQ }\end{array}$ \\
\hline & & & & & & \\
$\mathbf{0}$ & 24 & 0 & 0 & 0 & 3.1 & 20.9 \\
$\mathbf{1}$ & 12 & 9 & 1 & 12.5 & 6 & 23.3 \\
$\mathbf{2}$ & 22 & 16 & 8 & 24.3 & 11.8 & 27 \\
$\mathbf{3}$ & 15 & 12 & 7 & 28.3 & 15 & 25.6 \\
$\mathbf{4}$ & 19 & 18 & 13 & 46.4 & 15 & 29.4 \\
$\mathbf{5}$ & 15 & 13 & 9 & 44.3 & 21.9 & 27.8 \\
$\mathbf{6}$ & 13 & 13 & 9 & 47.5 & 20.6 & 28.6 \\
$\mathbf{7}$ & 6 & 6 & 4 & 62 & 17.6 & 39.3 \\
$\mathbf{8}$ & 5 & 4 & 3 & 42.2 & 24.9 & 26.4 \\
$\mathbf{9}$ & 4 & 4 & 3 & 55.8 & 26.4 & 35.2 \\
$\mathbf{1 0}$ & 4 & 4 & 4 & 49.5 & 39.4 & 33.5 \\
$\mathbf{1 1}$ & 1 & 1 & 1 & 59 & 38.6 & 43 \\
& & & & & & \\
\hline
\end{tabular}


Tableau III. Analyse de la variance (test F) de la clinique post-traumatique (scores moyens de la CAPS, de la DES, et du SDQ) en liaison avec le type d'événements vécus potentiellement traumatiques.

\begin{tabular}{|c|c|c|c|c|c|c|c|}
\hline \multicolumn{8}{|c|}{$\mathbf{F}(\mathbf{p})$} \\
\hline & Type d'évènements vécus & Scores moyens CA & PS & Scores moyens I & & Scores moyens $S$ & DQ \\
\hline 1. & Catastrophe naturelle & $1.87(\mathrm{p}<0.005)$ & $*$ & $1.28(\mathrm{p}=0.16)$ & $\mathrm{ns}$ & $2.30(\mathrm{p}<0.002)$ & * \\
\hline 2. & Incendie ou explosion & $1.68(\mathrm{p}<0.02)$ & $*$ & $1.20(\mathrm{p}=0.23)$ & ns & $1.48(\mathrm{p}=0.78)$ & ns \\
\hline 3. & Accident de transport & $1.42(\mathrm{p}=0.07)$ & $\mathrm{ns}$ & $1.19(\mathrm{p}=0.24)$ & $\mathrm{ns}$ & $0.94(\mathrm{p}=0.56)$ & ns \\
\hline 4. & Accident de travail ou domestique graves & $2.10(\mathrm{p}<0.001)$ & $*$ & $1.12(\mathrm{p}=0.32)$ & $\mathrm{ns}$ & $1.02(\mathrm{p}=0.44)$ & ns \\
\hline 5. & Exposition à une substance toxique dangeureuse & $2.30(\mathrm{p}<0.0003)$ & $*$ & $2.82(\mathrm{p}<0.0001)$ & $*$ & $6.14\left(\mathrm{p}<10^{6}\right)$ & * \\
\hline 6. & Agression physique & $2.06(\mathrm{p}<0.002)$ & $*$ & $2.27(\mathrm{p}<0.0005)$ & $*$ & $2.21(\mathrm{p}<0.002)$ & $*$ \\
\hline 7. & Agression avec une arme & $1.73(\mathrm{p}<0.02)$ & $*$ & $1.91(\mathrm{p}<0.005)$ & $*$ & $1.32(\mathrm{p}=1.56)$ & ns \\
\hline 8. & Agression sexuelle & $3.90(\mathrm{p}<0.000001)$ & $*$ & $2.01(\mathrm{p}<0.003)$ & $*$ & $2.30(\mathrm{p}<0.0001)$ & $*$ \\
\hline 9. & Autre expérience sexuelle pénible & $2.22(\mathrm{p}<0.0005)$ & $*$ & $2.50(\mathrm{p}<0.0002)$ & $*$ & $2.23(\mathrm{p}<0.002)$ & * \\
\hline 10. & Combat ou exposition à une zone en guerre & $0.94(\mathrm{p}=0.60)$ & ns & $1.13(\mathrm{p}=0.30)$ & $\mathrm{ns}$ & $0.87(\mathrm{p}=0.65)$ & ns \\
\hline 11. & Captivité & $2.99(\mathrm{p}<0.00001)$ & $*$ & $2.50(\mathrm{p}<0.0002)$ & $*$ & $2.42(\mathrm{p}<0.0006)$ & * \\
\hline 12. & Maladie ou blessure menaçant la survie & $1.96(\mathrm{p}<0.003)$ & $*$ & $1.69(\mathrm{p}<0.02)$ & * & $1.15(\mathrm{p}=0.30)$ & $\mathrm{ns}$ \\
\hline 13. & Souffrance humaine grave & $1.56(\mathrm{p}<0.04)$ & * & $2.25(p<0.0006)$ & * & $1.84(\mathrm{p}<0.02)$ & $*$ \\
\hline 14. & Mort violente (homicide, suicide) & $1.45(\mathrm{p}=0.06)$ & $\mathrm{ns}$ & $1.99(\mathrm{p}<0.003)$ & * & $1.67(\mathrm{p}<0.04)$ & $*$ \\
\hline 15. & Mort soudaine, inattendue d'un proche & $2.07(\mathrm{p}<0.002)$ & $*$ & $1.64(\mathrm{p}<0.03)$ & $*$ & $1.48(\mathrm{p}=0.08)$ & $\mathrm{ns}$ \\
\hline 16. & Blessure grave causée à quelqu'un d'autre & $2.19(\mathrm{p}=0.36)$ & $\mathrm{ns}$ & $1.85(\mathrm{p}<0.007)$ & $*$ & $1.58(\mathrm{p}=0.19)$ & ns \\
\hline
\end{tabular}

*=statistiquement significatif; $n s=$ non significatif 
Tableau IV. Scores moyens à la DES et au SDQ en fonction du diagnostic de PTSD actuel et par le passé.

\begin{tabular}{|c|c|c|c|c|}
\hline & \multicolumn{2}{|c|}{ PTSD actuel } & \multicolumn{2}{|c|}{ PTSD par le passé } \\
\hline & Présent $(n=62)$ & Absent $(n=78)$ & Présent (n=57) & Absent $(n=83)$ \\
\hline \multirow{2}{*}{ Scores moyens à la DES } & $\mathbf{2 3 . 0 3 4}$ (ET 14.556) & 7.921 (ET 8.474) & 21.529 (ET 13.924) & 9.865 (ET 11.524) \\
\hline & \multicolumn{2}{|c|}{ différence significative $(\mathrm{p}<0.05)$} & \multicolumn{2}{|c|}{ différence significative $(\mathrm{p}<0.05)$} \\
\hline \multirow{2}{*}{ Scores moyens au SDQ } & 32.097 (ET 10.209) & 23.115 (ET 4.431) & 31.737 (ET 9.793) & 23.904 (ET 6.258) \\
\hline & différence sigr & ative $(\mathrm{p}<0.05)$ & \multicolumn{2}{|c|}{ différence significative $(\mathrm{p}<0.05)$} \\
\hline
\end{tabular}

(ET=Ecart-Type) 